\title{
FMT for Severe C. difficile Infection: If at First You Do No Harm, the Second Time's a Charm
}

\author{
Ari Grinspan ${ }^{1}$ \\ Published online: 14 May 2020 \\ ○) Springer Science+Business Media, LLC, part of Springer Nature 2020
}

Over the past 10 years, there has been a substantial increase in the use of fecal microbiota transplantation (FMT) to treat recurrent Clostridioides difficile infection (CDI) after failed antibiotic therapy. Most medical societies across the world have incorporated FMT into their guidelines in recognition of the effectiveness of this treatment for recurrent CDI. The global cure rate of CDI after FMT is reported to be between 80 and $90 \%$. The clinical quandary is what to do with the $10-20 \%$ of patients that fail a first FMT. While there have been several studies exploring the risk factors for FMT failure such as severe/fulminant infection, inpatient status at time of FMT, and multiple CDI-related hospitalizations, there are no clear guidelines on how to proceed after a patient fails an initial FMT $[1,2]$.

Despite lack of guidelines, there has been growing evidence to support the efficacy of repeat FMT in these patients. Several retrospective studies have shown that a second FMT can be effective in patients who fail an initial FMT [3, 4]. When thinking about patients who fail FMT, it is important to categorize them into early and late failures [5]. There are likely different mechanisms of FMT failure, and understanding this can allow practitioners to better counsel their patients on the likelihood of needing additional FMTs. For example, severe or fulminant CDI should be viewed as a different phenotype of disease than recurrent CDI managed in the outpatient setting. In fact, Dr. Fischer and colleagues have shown that using a sequential FMT treatment algorithm for inpatients admitted with severe or fulminant CDI can be lifesaving [6]. The bottom line is that in clinical practice, it is common for practitioners to proceed with a second FMT, but there has been little to no data predicting who will fail this second FMT.

Ari Grinspan

ari.grinspan@mountsinai.org

1 The Henry D. Janowitz Division of Gastroenterology, Icahn School of Medicine at Mount Sinai, New York, NY, USA
In this issue of Digestive Disease and Sciences, Allegretti et al. [7] present a multicenter retrospective study of patients undergoing at least one FMT for CDI, specifically examining risk factors for patients who fail two FMTs. Over a 4-year period, the authors collected data on 540 patients finding an $80 \%$ primary cure rate. This cure rate is on the low end of what has been published previously and may reflect the number of high-risk patients included in their study. In their final analysis, they included 492 patients. A total of 63 patients underwent a second FMT: 36 (57\%) were successfully treated, 24 (38\%) failed, and $3(5 \%)$ were lost to follow up. Thus, 24 of the original $492(5 \%)$ failed a second FMT. They compared these 24 patients who failed 2 FMTs to all patients who were successfully treated by 1 or 2 FMTs. They identified three significant risk factors for failing 2 FMTs: immunocompromised status, inpatient status, and the presence of pseudomembranous colitis all at the time of the first FMT. By including patients who were successfully treated by 1 FMT in the comparator group, the authors did not directly evaluate the risk factors for failing a second FMT if a patient has already failed an initial FMT. Nevertheless, this study does provide framework guidance to discuss with patients before they undergo an initial FMT.

Approximately half of the patients who received a second FMT were those suffering from severe and fulminant disease. It is known from prior work that these patients are already at high risk of failing a single FMT [1]. Several pioneers in the field have established a protocol for treating these patients with a response-guided sequential FMT algorithm [6]. In the presence of pseudomembranous colitis, the protocol calls for an additional FMT to be performed 3-5 days later and repeating FMT until pseudomembranous colitis resolves. This current study confirms that the patients with severe or fulminant disease and pseudomembranous colitis are at risk of failing a second FMT. In my clinical practice, I have treated multiple patients with severe/fulminant CDI using this protocol. I have found that some patients require three or more FMTs to achieve clinical cure. 
The authors also found that immunocompromised status is a risk factor for failing multiple FMTs. In contrast, a recent meta-analysis showed that immunocompromised status was not a risk factor for failing an initial FMT [8]. It remains unclear as to why immunosuppressed patients would be at risk of failing a second FMT if they are not at risk of failing an initial FMT. There has been debate whether comorbid IBD decreases the efficacy of FMT for CDI. While the authors did not fully divulge the etiology of immunosuppression in this current study, IBD itself was not identified as a risk factor. While knowledge of patients' immune status is helpful when performing FMT, further studies are needed to determine its impact on multiple FMT failure.

All of these patients had FMT delivered during colonoscopy. The advantage of this approach is direct visualization of the colonic mucosa and assessment for pseudomembranous colitis-which this study found to be a risk factor for failing 2 FMTs. The disadvantages of colonoscopic delivery are invasiveness and cost. At this time, colonoscopic delivery is the most common method to deliver FMT, at least in the US. This trend will likely change in the future with the advent of capsule formulation, which has already been shown to be comparable to colonoscopic delivery [9, 10]. It is unknown if the risks for failing multiple FMTs as outlined in this study will also pertain to capsule delivery. As the landscape of FMT slowly evolves toward encapsulated oral products, we will have to reassess risk factors with additional studies.

This is the first study to specifically look at patients refractory to two FMTs - a thankfully small (5\%) but very challenging cohort of patients. While highly successful, FMT is not perfect. Knowing these risk factors should help practitioners shape a framework or strategy for how to approach and counsel these high-risk patients. This does not mean that practitioners should withhold FMT from these patients. In this study, more than half of the patients who needed 2 FMTs were cured, suggesting that a second FMT is a reasonable clinical strategy if the patient should initially fail. The main take home message is that practitioners should warn high-risk patients that two or more FMTs might be required to eradicate the infection. This study highlights the need for defined FMT protocols for managing high-risk patients. While multiple FMTs may be needed in certain populations, such as severe and fulminant CDI, further prospective studies are needed to define best practices.

\section{References}

1. Fischer M, Kao D, Mehta SR, et al. Predictors of early failure after fecal microbiota transplantation for the therapy of Clostridium difficile infection: a multicenter study. Am J Gastroenterol. 2016;111:1024-1031.

2. Patron RL, Hartmann CA, Allen S, et al. Vancomycin taper and risk of failure of fecal microbiota transplantation in patients with recurrent Clostridium difficile infection. Clin Infect Dis. 2017;65:1214-1217.

3. Agrawal M, Aroniadis OC, Brandt LJ, et al. The long-term efficacy and safety of fecal microbiota transplant for recurrent, severe, and complicated Clostridium difficile infection in 146 elderly individuals. J Clin Gastroenterol. 2016;50:403-407.

4. Perler BK, Chen B, Phelps E, et al. Long-term efficacy and safety of fecal microbiota transplantation for treatment of recurrent Clostridioides difficile infection. J Clin Gastroenterol. 2020. https ://doi.org/10.1097/MCG.0000000000001281.

5. Allegretti JR, Allegretti AS, Phelps E, et al. Classifying fecal microbiota transplantation failure: an observational study examining timing and characteristics of fecal microbiota transplantation failures. Clin Gastroenterol Hepatol. 2018;16:1832-1833.

6. Fischer M, Sipe BW, Rogers NA, et al. Faecal microbiota transplantation plus selected use of vancomycin for severe-complicated Clostridium difficile infection: description of a protocol with high success rate. Aliment Pharmacol Ther. 2015;42:470-476.

7. Allegretti JR, Mehta SR, Kassam Z, et al. Risk factors that predict the failure of multiple fecal microbiota transplantations for Clostridioides difficile infection. Dig Dis Sci. (Epub ahead of print). https://doi.org/10.1007/s10620-020-06198-2.

8. Shogbesan O, Poudel DR, Victor S, et al. A systematic review of the efficacy and safety of fecal microbiota transplant for Clostridium difficile infection in immunocompromised patients. Can J Gastroenterol Hepatol. 2018;2018:1394379.

9. Ramai D, Zakhia K, Fields PJ, et al. Fecal microbiota transplantation (FMT) with colonoscopy is superior to enema and nasogastric tube while comparable to capsule for the treatment of recurrent Clostridioides difficile infection: a systematic review and metaanalysis. Dig Dis Sci.. 2020. https://doi.org/10.1007/s10620-02006185-7.

10. Luo Y, Lucas AL, Grinspan AM. Fecal transplants by colonoscopy and capsules are cost-effective strategies for treating recurrent Clostridioides difficile infection. Dig Dis Sci. 2020;65:1125-1133.

Publisher's Note Springer Nature remains neutral with regard to jurisdictional claims in published maps and institutional affiliations. 